\title{
Strategies de Co-Opetition et Performance a l'export : Cas Des PME Marocaines Exportatrices
}

\author{
Mohamed Anis Tamiri, \\ Professeur de l'Enseignement Supérieur Assistant \\ Faculté des Sciences Juridiques Economiques et Sociales Ain Sebâa, \\ Université Hassan II de Casablanca, Maroc \\ Mina Elgraini, \\ Professeure de l'Enseignement Supérieur \\ Faculté des Sciences Juridiques Economiques et Sociales Souissi, \\ Université Mohammed V de Rabat, Maroc
}

Doi:10.19044/esj.2020.v16n25p88～URL:http://dx.doi.org/10.19044/esj.2020.v16n25p88

\begin{abstract}
Résumé
Les alliances stratégiques sont caractérisées par la simultanéité de la coopération et de la concurrence. Ce phénomène a été popularisé sous la notion de co-opétition. Le présent article s'intéresse à l'étude de l'impact des stratégies de co-opétition sur la performance export des PME. Les données collectées par questionnaire auprès d'un échantillon de 150 PME marocaines exportatrices ont fait l'objet d'une analyse par les équations structurelles selon l'approche PLS. Les résultats montrent que la confiance intervient comme modérateur de la relation de coopération entre concurrents. Ces derniers développent leur comportement concurrentiel pour atteindre leurs objectifs à l'export d'autant plus que la coopération existe et un climat de confiance s'instaure.
\end{abstract}

Mots clés : Co-opétition, Performance export, Confiance, Alliances stratégiques, PME exportatrices 


\title{
Co-Opetition Strategies and Export Performance : Case of Moroccan Exporting SME's
}

\author{
Mohamed Anis Tamiri, \\ Professeur de l'Enseignement Supérieur Assistant \\ Faculté des Sciences Juridiques Economiques et Sociales Ain Sebâa, \\ Université Hassan II de Casablanca, Maroc \\ Mina Elgraini, \\ Professeure de l'Enseignement Supérieur \\ Faculté des Sciences Juridiques Economiques et Sociales Souissi, \\ Université Mohammed V de Rabat, Maroc
}

\begin{abstract}
Strategic alliances are characterized by the simultaneity of cooperation and competition. This phenomenon was popularized under the notion of coopetition. This article focuses on the study of the impact of co-opetition strategies on the export performance of SMEs. The data collected by questionnaire from a sample of 150 Moroccan exporting SMEs were analyzed by structural equations according to the PLS approach. The results show that trust acts as a moderator of the cooperative relationship between competitors. The latter develop their competitive behavior to achieve their export objectives, especially as there is cooperation and a climate of trust is established.
\end{abstract}

Keywords: Co-opetition, Export performance, Trust, Strategic alliances, Exporting SME's

\section{Introduction}

L'intérêt porté par les économistes au phénomène d'internationalisation des entreprises date des années soixante. Il a occupé une place importante dans les travaux de recherche sur le commerce international. Les auteurs considèrent que l'export comme forme d'engagement sur un marché étranger est une première étape vers l'internationalisation (Rutashobya et Jaensson, 2004 ; Ruzzier et al., 2006).

Pour faire face aux exigences accrues d'adaptabilité à la mondialisation des échanges, plusieurs entreprises exportent dans le cadre d'alliances dîtes «stratégiques ». Ces dernières, à la différence des autres 
formes de partenariats, sont constituées par des concurrents qui optent pour la coopération inter organisationnelle.

La simultanéité de la coopération et de la concurrence est rendue ainsi possible grâce au développement d'alliances entre firmes concurrentes (Bengtsson et Kock, 2000 ; Tsai, 2002 ; Luo, 2004 ; Luo, 2005). Ce comportement «hybride» (Walley, 2007) a été popularisé par la notion de «co-opétition » grâce à Brandenburger et Nalebuff (1996). Ces derniers expliquent que les firmes combinent leurs forces dans l'objectif de gagner la plus grande part du « gâteau » (Brandenburger et Nalebuff, 1996). Dans un premier temps, elles coopèrent pour faire face à la concurrence avant de développer des actions concurrentielles pour s'accaparer une part de la valeur créée (Brandenburger et Nalebuff, 1996).

Dans leur revue de littérature portant sur les déterminants de la performance export, Sousa et al. (2008) recommandent de prendre en considération l'aspect relationnel et son impact sur la performance export. Ils proposent également de vérifier l'existence d'effets directs ou indirects sur la performance export en vérifiant l'effet modérateur ou médiateur de certaines variables (Zou et Stan, 1998).

Bensebaa (2003) recommande également de prendre en considération la dynamique de concurrence au sein des réseaux d'entreprises où les relations inter organisationnelles sont caractérisées par la coopération et la concurrence simultanées.

Le présent article est une réponse à ces perspectives de recherche. Son objet de recherche est formulé comme suit :

\section{Dans quelle mesure les stratégies de co-opétition contribuent-elles à la performance export des PME marocaines?}

Pour répondre à cette problématique, une revue de la littérature a permis de dresser un état des lieux des approches de l'internationalisation des entreprises et de la recherche sur les difficultés d'exportation chez les PME. Ensuite, les avantages et la problématique des alliances startégiques, à savoir le phénomène de co-opétition et ses composantes, sont abordés. Enfin, sont présentés la démarche méthodologique, l'analyse des données et la discussion des résultats de l'enquête.

\section{Les approches de l'internationalisation des entreprises :}

L'examen de la littérature fait apparaitre trois grilles d'analyse du phénomène d'internationalisation des entreprises : 1/ l'école comportementale ou béhaviorale qui trouve ses fondements dans l'approche de l'internationalisation par les étapes, 2/ l'école des alliances développée grâce à l'approche par les réseaux et enfin, 3/ l'école de la pensée économique fondée sur les théories des investissements directs étrangers. 


\subsection{L'approche par les étapes :}

L'école comportementale trouve ses fondements dans le modèle Uppsala développé par Johanson et Weidersheim-Paul (1975) puis formulé par Johanson et Valhne (1990). Ce modèle constitue le cadre d'analyse le plus utilisé pour décrire et expliquer le phénomène d'internationalisation des entreprises.

A partir d'une étude réalisée sur quatre firmes suédoises, Johanson et Weidersheim-Paul (1975) concluent que le processus d'internationalisation des entreprises est une succession d'étapes. Dans un premier temps, elles décident de se lancer sur des marchés étrangers à travers des activités d'exportation, poursuivent leur progression par la création de filiales à l'étranger pour passer en dernier lieu à la délocalisation de leurs unités de production (Johanson et Weidersheim-Paul, 1975).

Les auteurs expliquent également que la distance psychologique qui sépare l'entreprise de son marché étranger diminue d'autant plus que le dirigeant et son organisation acquièrent de l'expérience à l'international (Johanson et Valhne, 1990). Ainsi, pour réussir leur processus d'internationalisation, les entreprises ont intérêt à se lancer sur des marchés «psychologiquement» proches de leur marché domestique (Ramadan et Levratto, 2011).

Pour combler les insuffisances théoriques de cette approche et mieux décrire le phénomène d'internationalisation des entreprises, un réexamen du modèle initial de l'école suédoise par ses fondateurs même a permis de développer une nouvelle conception de l'internationalisation, celle de l'approche par les réseaux qui constitue un prolongement de l'approche par les étapes (Johanson et Vahlne, 1990).

\subsection{L'approche par les réseaux :}

L'école des alliances décrit l'internationalisation comme un processus de développement d'une entreprise avec un ou plusieurs acteurs sur les marchés étrangers (Johanson et Vahlne, 1990). Les relations inter organisationnelles permettent de mutualiser les forces et de faciliter l'accès aux ressources nécessaires à la croissance d'une organisation ainsi qu'à son développement à l'international (Li et al., 2004).

Grâce à la complémentarité comme mécanisme d'implication des acteurs, la coopération inter organisationnelle permet un partage des risques et une accélération du processus d'internationalisation. (Chtourou, 2005).

\subsection{L'approche économique :}

L'école de la pensée économique passe par les théories du commerce international, en particulier la théorie des investissements directs étrangers (IDE). Selon cette approche, l'entreprise s'internationalise sans avoir à 
accumuler une expérience sur des marchés étrangers. La participation au capital d'une entreprise étrangère est le mode d'internationalisation le plus sûr (Ramadan et Levratto, 2011).

La rapidité, la minimisation du risque d'échec et la volonté de l'investisseur à contourner les barrières à l'entrée (Ramadan et Levratto, 2011), sont les principales raisons derrière le choix de cette stratégie de croissance et/ou d'internationalisation.

\section{PME et difficultés à l'export :}

L'export est considéré comme une première étape vers l'internationalisation (Ruzzier et al., 2006). Ses avantages, tant pour l'entreprise que pour l'économie d'un pays, sont partout reconnus.

Un examen de la littérature montre que les PME éprouvent des difficultés en raison de leur taille. L'engagement à l'export s'avère une décision difficile à prendre par les PME en raison des obstacles qu'elles doivent surmonter.

Selon Leonidou et Katsikeas (1996), un obstacle à l'export est « tout obstacle d'attitude, de structure, opérant, ou toute autre entrave rendant difficile ou freinant la volonté de l'entreprise de commencer, de développer ou de maintenir une activité internationale ».

Arteaga et Fernandez (2008) proposent de regrouper les barrières à 1'export recensées à travers une revue de littérature en quatre catégories : 1/ Les barrières liées à la connaissance ou barrières liées au manque d'informations ainsi qu'à la méconnaissance des fondamentaux d'une activité d'exportation 2/ Les barrières liées aux moyens 3/ Les barrières liées au processus 4/ Les barrières exogènes.

\subsection{Les barrières liées à la connaissance :}

Plusieurs travaux confirment l'effet positif de la connaissance des marchés étrangers sur l'engagement à l'export. Selon Aharoni (1966), la méconnaissance des marchés étrangers empêche les organisations à se lancer dans des activités au delà des frontières de leur lieu d'implantation.

\subsection{Les barrières liées aux moyens :}

L'absence de moyens nécessaires pour affronter les difficultés rencontrées empêche l'accès aux marchés étrangers. Les travaux de Katsikeas et al. (2000) montrent que le manque de capacités de production constitue pour l'entreprise un obstacle lors de la phase de mise en œuvre et de développement d'une activité d'exportation. 


\subsection{Les barrières liées au processus :}

Il s'agit d'obstacles rencontrés à l'occasion de l'exercice d'une activité d'exportation. Comme exemples, on peut citer la complexité de la documentation et des démarches liées aux opérations d'exportation, les moyens logistiques et le coût élevé de transport etc... (Arteaga et Fernandez, 2008).

\subsection{Les barrières exogènes :}

Ces barrières sont incontrôlables car elles proviennent de l'action des autres intervenants sur les marchés étrangers. La recherche sur les barrières exogènes montre que la concurrence sur le marché étranger, le risque de change et l'instabilité politique chez certains pays étrangers constituent les principales barrières sur lesquelles l'entreprise exportatrice n'a aucun pouvoir de contrôle (Arteaga et Fernandez, 2008).

\section{Les alliances stratégiques : Définition et avantages}

Dussauge et Garrette (1991) définissent les alliances stratégiques comme «Des partenariats entre plusieurs entreprises concurrentes, ou potentiellement concurrentes, qui choisissent de mener à bien un projet ou une activité spécifique en coordonnant les compétences, moyens et ressources nécessaires plutôt que de se faire concurrence sur l'activité concernée, ou de fusionner entre elles ou de procéder à des cessions ou acquisitions d'activité ».

Cette forme de partenariat inter ogranisationnel permet de conduire une action commune tout en restant stratégiquement autonome et juridiquement indépendant (Jolly, 2001). Les alliés définissent leur stratégie, s'engagent à long terme dans une action conjointe, assument collectivement les risques et partagent les responsabilités ainsi que le pouvoir décisionnel.

L'alliance permet également la mise en commun de ressources, tangibles et/ou intangibles, pouvant servir les alliés pour détenir un avantage concurrntiel (Barney, 1991). Grâce à la coopération, les alliés accèdent à des ressources pour lesquels ils ne pouvaient prétendre de manière individuelle.

Enfin, il est à souligner que les avantages de la coopération dépendent à la fois des actions de la firme et de celles de son/ses allié (s), en d'autres termes, du niveau d'interdépendance au sein de l'alliance : Les alliés ont intérêt à ce que l'alliance se prolonge d'autant plus que le degré d'interdépendance est élevé (Trabelsi, 2007).

\section{Problématique des alliances : La co-opétition : Définitions, avantages et risques}

Plusieurs auteurs ont remarqué l'existance de partenariats entre concurrents. Cela montre que les comportements de coopération et de 
concurrence peuvent être simultanés. La concurrence trouve sa légitimité dans la divergence des intérêts alors que la coopération fait appel aux capacités collaboratives des acteurs en raison de la convergence de leurs intérêts (Dagnino et al., 2007). Ce comportement « hybride » a été popularisé à travers la notion de «co-opétition » (Brandenburger et Nalebuff, 1996).

La co-opétition permet de combiner les avantages de la coopération et ceux de la concurrence. Elle intervient pour favoriser une situation gagnantgagnant à travers la mutualisation des forces, le partage des risques et la réduction globale des coûts (Bouncken et al., 2015).

\subsection{Définitions de la co-opétition :}

Brandenburger et Nalebuff (1996) étaient les premiers à avoir défini la co-opétition comme "un rapprochement d'intérêts entre complémenteurs lorsque la coopération et la compétition se produisent simultanément ». Un examen de la littérature fait apparaitre d'autres définitions :

Tableau 1 : Reproduit à partir d'une revue de littérature

\begin{tabular}{|c|l|}
\hline Auteurs & \multicolumn{1}{|c|}{ Définitions de la Co-opétition } \\
\hline $\begin{array}{c}\text { Bengtsson et Kock } \\
(2000)\end{array}$ & $\begin{array}{l}\text { «La co-opétition est une relation dyadique et paradoxale qui } \\
\text { émerge quand deux entreprises coopèrent dans quelques } \\
\text { activités et qui sont en même temps en compétition l'une avec } \\
\text { l'autre sur d'autres activités » }\end{array}$ \\
\hline $\begin{array}{c}\text { Dagnino et Padula } \\
\text { (2002) }\end{array}$ & $\begin{array}{l}\text { "La co-opétition est une stratégie inter-entreprises où les } \\
\text { concurrents s'engagent dans des intérêts partiellement } \\
\text { convergents, dans une structure, dans le but de créer de la } \\
\text { valeur par des avantages coopératifs » }\end{array}$ \\
\hline $\begin{array}{c}\text { Dagnino, Le Roy et } \\
\text { Yami (2007) }\end{array}$ & $\begin{array}{l}\text { "La co-opétition est un système d'acteurs qui interagissent } \\
\text { sur la base d'une congruence partielle des intérêts et des } \\
\text { objectifs» }\end{array}$ \\
\hline
\end{tabular}

Bouncken et al. (2015) ont proposé une définition selon laquelle «la co-opétition est un processus stratégique et dynamique dans lequel les acteurs économiques créent conjointement de la valeur à travers une relation d'interaction coopérative, pendant qu'ils concurrencent simultanément pour capter une partie de cette valeur». Ainsi, on comprend qu'il s'agit d'une stratégie qui consiste en la présence simultanée des comportements de coopération et de concurrence inter organisationnels.

\subsection{Avantages de la co-opétition :}

Les entreprises qui opèrent sur un même marché, répondent aux mêmes besoins clients et affrontent les mêmes risques, ont intérêt à opter pour une stratégie de co-opétition vue les multiples avantages qu'elle procure (Bouncken et al. 2015).

La recherche sur ce phénomène montre sa pertinence en matière de partage des coûts, par conséquent une économie d'échelle en faveur des 
acteurs impliqués. La co-opétititon permet également de faire face aux changements incessants, à l'instabilité et à l'incertitude qui caractérisent les activités économiques, par conséquent une réduction des risques (Gnyawali, 2011).

En matière d'innovation, les études montrent que la co-opétition présente l'avantage de surmonter les asymétries de connaissances puisque certains concurrents détiennent des connaissances qu'il est intéressant de mettre en commun pour lancer de nouveaux produits (Ritala, 2012).

\subsection{Risques liés à la co-opétition :}

En raison de la nature paradoxale de l'alliance constituée par deux ou plusieurs entreprises juridiquement indépendantes, la co-opétition est une stratégie à haut niveau de risque (Fernandez et Le Roy, 2010).

Lorsqu'une entreprise décide de coopérer avec son concurrent, elle augmente la probabilité que ses ressources soient identifiées et imitées. Des tensions peuvent prendre naissance du moment où les entreprises cherchent à accumuler le savoir-faire d'un autre concurrent tout en veillant à la protection de leur cœur de compétence (Fernandez et Le Roy, 2010).

Ces risques devront conduire au choix d'un mode organisationnel susceptible de réduire les coûts de transaction qu'une firme est appelée à supporter à l'occasion d'une coopération avec ses concurrents.

\section{La performance export : Définition et mesure}

Le nombre de travaux réalisés pour l'étude de la performance export témoignent de l'importance de la question et de sa légitimité comme objet de recherche. La première étude du rendement à l'export est attribuée à Tookey (1964) qui a cherché à identifier les facteurs clés de succès des entreprises à l'export. Par la suite, il a fallu attendre jusqu'aux années 80 pour assister à une émergence des études sur la performance export (Sousa et al., 2008).

Les auteurs se sont intéressés principalement aux déterminants internes, laissant de côté toute prise en compte des facteurs environnementaux et leur impact sur la performance export. Sousa et al. (2008) recommandent de prendre en considération l'aspect relationnel et son impact sur la performance export vue que les relations qu'entretient une entreprise avec ses fournisseurs, ses clients et ses concurrents, sont généralement fondées sur la coopération mutuelle et l'interdépendance.

L'enquête menée par Zou et Stan (1998) auprès d'un échantillon d'exportateurs américains et japonais a permis de tester et valider une échelle de mesure de la performance export : L'échelle EXPERF. Selon Zou et Stan (1998), il s'agit de «la performance financière et stratégique de l'entreprise à l'exportation et la satisfaction de l'entreprise vis-à-vis de ce projet d'exportation ». 


\subsection{La dimension financière de la performance export :}

La performance export doit faire l'objet d'une évaluation financière (Zou et Stan, 1998). En effet, réaliser des profits sur un marché, accroitre le volume des ventes etc... constituent souvent des objectifs financiers à atteindre par les entreprises exportatrices et/ou non exportatrices.

\subsection{La dimension stratégique de la performance export :}

En dehors des objectifs financiers, les entreprises exportatrices se fixent des objectifs stratégiques tels que l'amélioration de leur compétitivité, l'augmentation de leur part de marché et le renforcement de leur position concurrentielle (Zou et Stan, 1998). De ce fait, la dimension stratégique est un indicateur pertinent pour l'évaluation de la performance d'une entreprise sur un marché étranger.

\subsection{La satisfaction vis-à-vis de l'activité d'export :}

Zou et Stan (1998) estiment que la satisfaction est susceptible d'impliquer le management et par voie de conséquence se traduire en performance. De ce fait, la satisfaction constitue une mesure pertinente du rendement à l'export.

\section{Le comportement concurrentiel :}

Plusieurs auteurs on recommandé de s'intéresser à la dynamique de concurrence au sein des réseaux d'entreprises où les relations inter organisationnelles sont caractérisées par la coopération et la concurrence simultanées (Bensebaa, 2003; Sousa et al., 2008). La dynamique de concurrence sur un marché est définie comme «l'ensemble des actions et des réactions concurrentielles d'une entreprise » (Bensebaa, 2003).

Les auteurs considèrent que la performance de l'entreprise est la conséquence directe de ses actions concurrentielles (Bensebaa, 2000). De ce fait, une première hypothèse peut être formulée comme suit :

\section{H1 : Le comportement concurrentiel exerce un effet positif direct sur la performance export}

\section{La coopération inter organisationnelle :}

Dans son étude portant sur la coopération dans un réseaux de prestataires de services logistiques en Chine, Xiang (2013) distinguent entre la coopération en termes de ressources et la coopération en termes d'activités.

La coopération en termes de ressources est définie comme «une relation entre entreprises juridiquement indépendantes, par laquelle elles mettent en commun, partagent et échangent des ressources, en vue d'atteindre des objectifs communs » (Xiang, 2013), alors que la coopération en termes d'activités est «une relation entre des entreprises juridiquement 
indépendantes, par laquelle elles coordonnent des activités complémentaires ou identiques, en vue d'atteindre des objectifs communs » (Xiang, 2013).

La coopération inter organisationnelle permet aux alliés de poursuivre des objectifs communs. Elle semble donc exercer une influence sur leur performance export, d'où l'hypothèse suivante :

H2 : La coopération inter organisationnelle exerce un effet positif indirect sur la performance export

\section{Comportement concurrentiel et coopération inter organisationnelle : Quelle relation ?}

Grâce à la coopération, les entreprises ont accès à des ressources tangibles et intangibles qui détermineront par la suite leurs capacités compétitives sur le marché (Barney, 1991). Un examen de la littérature montre l'existence de deux approches théoriques contradictoires.

La première suppose que plus l'entreprise est impliquée dans son réseau en vue d'accéder à des ressources nouvelles grâce à la coopération, plus elle est amenée à modérer son comportement agressif à l'égard des acteurs qui lui fournissent des ressources (Porter et Fuller, 1986). Cela s'explique par sa dépendance vis-à-vis de ses partenaires. De ce fait, une hypothèse peut être formulée comme suit :

H3 : Il existe une corrélation négative entre le comportement de coopération et le comportement concurrentiel

La deuxième approche soppose qu'un encastrement fort de la part d'une organisation conduit à renforcer son agressivité concurrentielle (Gnyawali et al., 2006). Dans ce cas, la coopération permet d'accéder à des ressources nouvelles et entraine en conséquence l'initiation d'actions concurrentielles. Il est donc possible de formuler une hypothèse comme suit :

H4 : Il existe une corrélation positive entre le comportement de coopération et le comportement concurrentiel

\section{La confiance : Définition, rôle et typologie}

L'examen de la littérature montre l'absence d'un véritable consensus quant à la définition de la confiance. Les travaux de Ramonjavelo et al (2006) ont permis de dinstinguer entre trois types de confiance : interpersonnelle, institutionnelle et inter organisationnelle.

La confiance « interpersonnelle » est la confiance que placent les individus dans d'autres. Elle s'explique par la réputation et/ou la compétence reconnues le plus souvent à l'occasion d'expériences antérieures et se traduit par la coopération et l'entraide des individus les uns avec les autres (Ramonjavelo et al., 2006). 
La confiance «Institutionnelle » dite également «Systémique » a été définie par Mangematin (1999) comme «Une autorité supérieure au sein de la société. Elle protège les individus ou les organisations contre les risques d'aléa moral ». Les structures sociales formelles constituent donc un garant des attributs d'un individu et/ou d'une organisation.

La confiance institutionnelle se développe grâce à l'acceptation et la reconnaissance des règles régissant la vie politique, économique et sociale, et se manifeste par le recours des parties contractantes à diverses institutions tels que les lois, les règlements et les contrats.

Enfin, la confiance «inter organisationnelle » qui régit les relations entre les organisations. Elle est fondée sur la réputation, la qualité des relations antérieures ou encore leur fréquence (Ramonjavelo et al.,2006).

Trabelsi (2007) estime que, dans le cadre d'une alliance, la confiance peut se construire à deux niveaux : au niveau des managers des firmes alliées en tant que confiance interpersonnelle et au niveau des entreprises partenaires en tant que confiance inter organisationnelle.

En raison de l'importance de la confiance dans les relations de coopération entre concurrents, il est possible de formuler une hypothèse comme suit :

\section{H5 : La confiance exerce un effet modérateur dans la relation de coopération entre concurrents}

\section{Démarche méthodologique de mise en œuvre de la recherche :}

Comme déjà souligné, l'objet de ce travail de recherche porte sur l'étude de l'impact des stratégies de co-opétition sur la performance export des PME. Les choix du terrain d'investigation et de la démarche méthodologique de mise en oeuvre de la recherche, sont d'une importance cruciale.

En raison de la sumiltanéité des comportement de coopération et de concurrence dans une relation de co-opétition, cette dernière est observée suivant ses deux dimensions : Coopération et Concurrence. Quant à la démarche méthodologique, elle est purement quantitaive. Cette dernière accorde une grande importance aux concepts et/ou variables grâce auxquels l'objet de recherche est à la fois schématisé et synthétisé de manière à donner une image réduite du phénomène étudié. L'objectif étant donné l'indépendance du chercheur ainsi que l'objectivité de ses découvertes. 


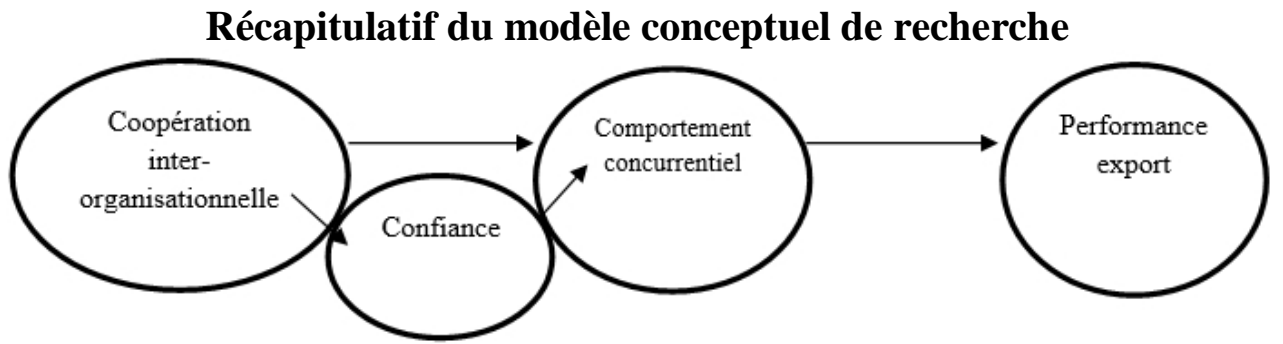

La collecte de données a nécessité l'administration d'un questionnaire auprès de PME constituant un groupement. Ce dernier prend généralement la forme juridique d'un groupement d'intérêt économique (GIE). L'échantillon final comprend 120 PME marocaines exportatrices membres d'alliances.

\section{Traitement et analyse de données :}

\subsection{Validation des instruments : $L$ 'analyse factorielle exploratoire}

Dans un premier temps, nous avons appliqué une analyse factorielle exploratoire aux données collectées afin de valider les instruments de mesure. Pour être valide, un instrument de mesure doit présenter un niveau de fiabilité et de validité satisfaisants.

Vernette (1991) décrit la fiabilité comme «le degré de reproductibilité des résultats lorsque l'on applique une nouvelle fois l'instrument dans des conditions identiques » alors que la validité d'un instrument de mesure permet de «s'assurer que ce qui est mesuré est bien le concept étudié » (Vernette, 1991).

Dans notre cas, la fiabilité des instruments de mesure est testée par le coefficient alpha de Cronbach ${ }^{4}$ alors que la validité a fait l'objet des tests $\mathrm{KMO}^{5}$ et de Sphéricité de Bartlett ${ }^{6}$.

\subsection{L'analyse confirmatoire par les équations structurelles}

Après validation des instruments de mesure, une analyse confirmatoire a été réalisée par le biais des équations structurelles (L'approche PLS) suivant quatre étapes : D'abord, un test de la validité convergente, ensuite un test de la validité discrminante, puis le test des hypothèses de recherche et enfin, un test de la capacité prédictive du modèle conceptuel.

${ }^{4}$ Le seuil admis est de 0.7 (Nunnally, 1978 ; Tenenhaus et al. 2005);

${ }^{5}$ Selon Malhotra, Décaudin \& Bouguera (2004), une valeur comprise entre 0,5 et 1 est largement acceptable ;

${ }^{6}$ Le test de sphéricité de Bartlett doit tendre vers. Cette valeur est pratiquement toujours satisfaite (Stewart, 1981 ; Evrard, Pras \& Roux, 2003) mais ce test reste sensible au nombre d'observations et presque toujours significatif lorsque l'échantillon est assez important (Pichon, 2006) 
La validité convergente repose sur l'examen des corrélations entre les mesures et leurs construits respectifs. En règle générale, les chercheurs considèrent que le coefficient de corrélation doit être supérieur à 0.7. Cela signifie qu'il y a plus de variance partagée entre le construit et sa mesure que d'erreur de variance (Fernandes, 2012).

Trois indicateurs permettent de tester la validité convergente: Loadings (Les charges externes), $\boldsymbol{C R}$ (La fiabilité composite) et l'A $\boldsymbol{V E}$ (La variance moyenne extraite). Les charges externes doivent être supérieures à 0.5 (Lacroux, 2010) mais, il est recommandé que les valeurs des charges externes soient supérieures à 0.7 (Balambo et Baz, 2014). La validité convergente a fait l'objet d'un deuxième test à savoir le $\boldsymbol{C R}$. Ce dernier doit être supérieur à 0.7 (Balambo et $\mathrm{Baz}, 2014)$. Enfin, un troisième indicateur à savoir l'A $\boldsymbol{V} \boldsymbol{E}$ dont la significativité implique que chaque indicateur standardisé partage plus de variance avec son construit latent qu'avec son erreur de mesure (Fornell et Larker, 1981 ; Lacroux, 2010). Il est recommandé que l'AVE soit supérieure ou égale à 0.5 .

La validité discriminante renvoie à la capacité des items d'un instrument de mesure à se distinguer suffisamment des items destinés à mesurer d'autres phénomènes voisins. Elle consiste à s'assurer que les items mesurant un construit sont fortement corrélés avec celui qu'ils sont censés mesurer, qu'avec les autres construits du même modèle (Lacroux, 2010).

Tableau 2 : Reproduit à partir des résultats de l'analyse factorielle exploratoire par le

logiciel SPSS

\begin{tabular}{|c|c|c|c|c|}
\hline $\begin{array}{c}\text { Construit (Ou } \\
\text { dimension du construit) }\end{array}$ & $\begin{array}{c}\text { Test de } \\
\text { KMO }\end{array}$ & $\begin{array}{c}\text { Test de } \\
\text { Sphéricité de } \\
\text { Bartlett }\end{array}$ & $\begin{array}{c}\text { Coefficient alpha } \\
\text { de Cronbach }\end{array}$ & Résultat \\
\hline Dimension financière & 0.749 & 0.000 & 0.731 & Instrument valide \\
\hline Dimension stratégique & 0.755 & 0.000 & 0.910 & Instrument valide \\
\hline $\begin{array}{c}\text { Satisfaction vis-à-vis du } \\
\text { projet d'exportation }\end{array}$ & 0.821 & 0.000 & 0.893 & Instrument valide \\
\hline $\begin{array}{c}\text { Irréversibilité des } \\
\text { actions }\end{array}$ & 0.637 & 0.000 & 0.843 & Instrument valide \\
\hline Confiance & 0.690 & 0.000 & 0.795 & Instrument valide \\
\hline $\begin{array}{c}\text { Coopération par des } \\
\text { ressources physiques }\end{array}$ & 0.743 & 0.000 & 0.913 & Instrument valide \\
\hline $\begin{array}{c}\text { Coopération par des } \\
\text { ressources } \\
\text { informationnelles }\end{array}$ & 0.593 & 0.000 & 0.919 & Instrument valide \\
\hline $\begin{array}{c}\text { Coopération par des } \\
\text { connaissances }\end{array}$ & 0.588 & 0.000 & 0.885 & Instrument valide \\
\hline
\end{tabular}


Tableau 3: Reproduit à partir des résultats des tests de validité convergente sur le logiciel Smart PLS

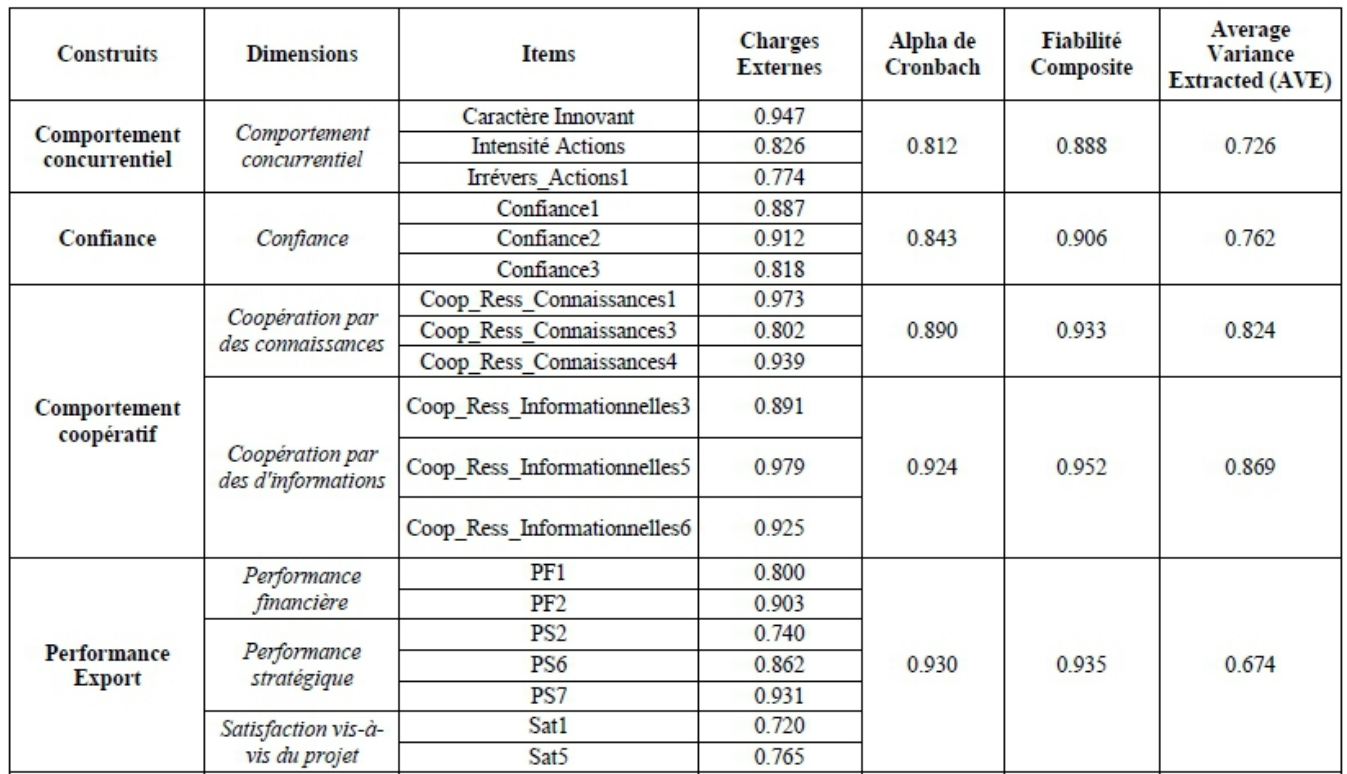

Tableau 4 : Reproduit à partir des résultats du test de la validité discriminante sur le logiciel Smart PLS

\begin{tabular}{|c|c|c|c|c|}
\hline & $\begin{array}{c}\text { Comportement } \\
\text { Concurrentiel }\end{array}$ & $\begin{array}{c}\text { Comportement } \\
\text { Coopératif }\end{array}$ & Confiance & $\begin{array}{c}\text { Performance } \\
\text { Export }\end{array}$ \\
\hline $\begin{array}{c}\text { Comportement } \\
\text { Concurrentiel }\end{array}$ & 0.852 & 0.906 & & \\
\hline $\begin{array}{c}\text { Comportement } \\
\text { Coopératif }\end{array}$ & -0.057 & 0.781 & 0.873 & \\
\hline $\begin{array}{c}\text { Confiance } \\
\text { Performance } \\
\text { Export }\end{array}$ & 0.234 & 0.600 & 0.837 & 0.821 \\
\hline
\end{tabular}


Tableau 5 : Reporoduit à partir des résultats des test d'hypothèses sur le logiciel Smart PLS

\begin{tabular}{|c|c|c|c|c|c|}
\hline & $\begin{array}{c}\text { Échantillon } \\
\text { initial (O) }\end{array}$ & $\begin{array}{c}\text { Moyenne de } \\
\text { l'échantillon } \\
(\mathrm{M})\end{array}$ & $\begin{array}{c}\text { Écart-type } \\
\text { (STDEV) }\end{array}$ & $\begin{array}{c}\text { Valeur t }(\mid \\
\text { O/STDEV |) }\end{array}$ & valeurs-p \\
\hline $\begin{array}{c}\text { Comportement Concurrentiel } \\
\text { >>> Performance Export }\end{array}$ & $\mathbf{0 . 4 0 3}$ & 0.429 & 0.045 & 8.968 & $\mathbf{0 . 0 0 0}$ \\
\hline $\begin{array}{c}\text { Comportement Coopératif } \\
\text { >>> Comportement } \\
\text { Concurrentiel }\end{array}$ & $\mathbf{- 0 . 6 1 4}$ & -0.615 & 0.170 & 3.609 & $\mathbf{0 . 0 0 0}$ \\
\hline $\begin{array}{c}\text { Comportement Coopératif } \\
>>>>\text { Confiance }\end{array}$ & $\mathbf{0 . 7 8 1}$ & 0.780 & 0.034 & 22.830 & $\mathbf{0 . 0 0 0}$ \\
\hline $\begin{array}{c}\text { Confiance >>> } \\
\text { Comportement Concurrentiel }\end{array}$ & $\mathbf{0 . 7 1 4}$ & 0.718 & 0.134 & 5.329 & $\mathbf{0 . 0 0 0}$ \\
\hline
\end{tabular}

Tableau 6 : Récapitulatif des résultats des tests d'hypothèses - Elaboré par les auteurs

\begin{tabular}{|c|l|c|}
\hline H1 & \multicolumn{1}{|c|}{ Hypothèses de recherche } & Résultats \\
direct sur la performance à l'export & Validée \\
\hline $\mathbf{H 2}$ & $\begin{array}{l}\text { La coopération inter organisationnelle exerce un effet } \\
\text { positif indirect sur la performance à l'export }\end{array}$ & Validée \\
\hline $\mathbf{H 3}$ & $\begin{array}{l}\text { Il existe une corrélation négative entre le comportement } \\
\text { de coopération et le comportement concurrentiel }\end{array}$ & Validée \\
\hline $\mathbf{H 4}$ & $\begin{array}{l}\text { Il existe une corrélation positive entre le comportement } \\
\text { de coopération et le comportement concurrentiel }\end{array}$ \\
\hline $\mathbf{H 5}$ & $\begin{array}{l}\text { La confiance exerce un effet modérateur dans la relation } \\
\text { de coopération entre concurrents }\end{array}$ & Validée \\
\hline
\end{tabular}

Tableau 7 : Reproduit à partir des résultats du test de la capacité prédictive du modèle conceptuel par le logiciel Smart PLS

\begin{tabular}{|l|c|}
\hline \multicolumn{1}{|c|}{ Variables à expliquer } & $\mathbf{R}^{\mathbf{2}}$ \\
\hline Comportement Concurrentiel & 0.202 \\
\hline Confiance & 0.610 \\
\hline Performance Export & 0.162 \\
\hline
\end{tabular}


Figure 1 : Modèle structurel - Reproduit à partir du logiciel Smart PLS

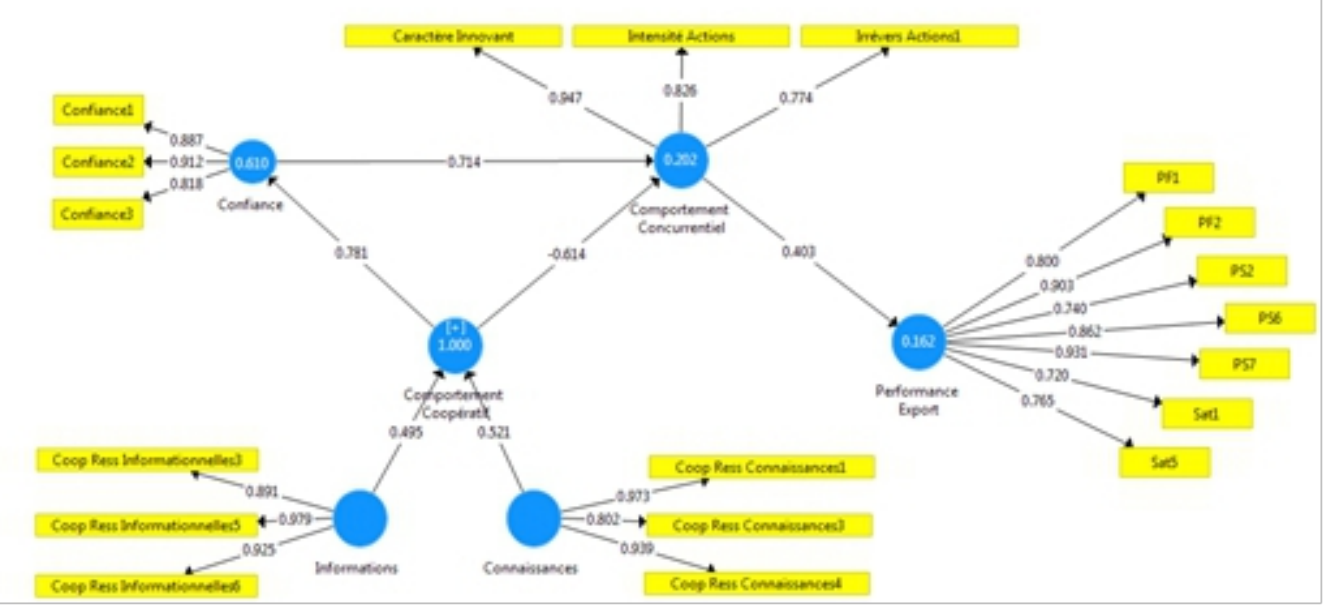

Toutes les valeurs des coefficients de détermination $\mathrm{R}^{2}$ sont supérieures à 0.1 , par conséquent le modèle structurel est significatif (Croutsche, 2002). Trois constats peuvent être dégagés :

- La variabilité de la performance export des PME marocaines dépend de leurs actions concurrentielles ;

- La variabilité du comportement concurrentiel des PME marocaines exportatrices dépend du niveau de confiance entre les membres de l'alliance ;

- La variabilité du niveau de confiance qui s'instaure entre les membres d'une alliance est fonction croissante de leur comportement de coopération inter organisationnelle.

\section{Discussion des résultats :}

Les résultats de l'étude montrent l'existence d'un effet positif et significatif de la coopération inter organisationnelle sur le niveau de confiance développée entre les membres du groupement. La coopération inter organisationnelle constitue un antécédent de la confiance. Le partage et/ou l'échange des informations sur les marchés étrangers, des connaissances et savoir-faire sur la pratique du commerce international, permet d'instaurer un climat de confiance entre les PME exportatrices en alliance.

Les résultats montrent également l'existence d'un effet positif et significatif de la confiance sur le comportement concurrentiel. En effet, la confiance intervient comme « lubrifiant» au sens d'Arrow (1968) afin que la coopération inter organisationnelle puisse aboutir à ses objectifs.

S'agissant de l'impact du comportement concurrentiel sur la performance export des PME, les résultats montrent l'existence d'un effet 
positif direct (Bensebaa, 2000). Ainsi, on comprend que les actions concurrentielles des PME exportatrices membres d'alliances permettent d'aboutir à leurs objectifs financiers, stratégiques, voire une satisfaction visà-vis de leur projet d'exportation.

L'utilisation de l'échelle EXPERF comme instrument de mesure de la performance export a permis de conclure que les dirigeants de PME marocaines exportatrices sont à la recherche d'objectifs financiers mais également stratégiques. Cela rejoint les conclusions de Zou et al. (1998) selon lesquelles les PME exportatrices se fixent des objectifs stratégiques, plus particulièrement l'amélioration de leur compétitivité, l'augmentation de leur part de marché et le renforcement de leur position concurrentielle, en plus de leurs objectifs financiers poursuivis à travers le commerce international. Ce résultat peut paraitre contradictoire avec la nature de la PME vue le nombre de travaux ayant montré l'absence d'une vision stratégique chez les petites et moyennes structures, ce qui est synonyme de dénaturation de la PME au sens de Torrès (2004).

Notre étude de la coopération entre PME exportatrices dans le cadre d'une alliance stratégique montre que ce comportement repose sur le partage et/ou l'échange d'informations concernant les marchés étrangers et les opportunités d'affaires qui s'offrent à l'international, la définition et la planification des activités d'exportation, en plus du savoir-faire dans le domaine tiré de la formation ou des expériences vécues.

La coopération inter organisationnelle est un comportement stratégique pour la PME exportatrice grâce à laquelle, et sous l'effet modérateur de la confiance, les alliés deviennent capables de développer des actions concurrentielles ce qui leur permet un accès aux marchés étrangers et de réaliser leurs objectifs à l'international. La coopération trouve sa légitimité dans leur besoin en ressources « stratégiques » (Barney, 1991) et se manifeste par un sentiment de confiance qui se développe au sein de l'alliance.

Enfin, cette étude montre que la probabilité qu'une activité d'exportation soit développée d'autant plus que la taille de l'entreprise est grande, n'est pas une hypothèse valide.

\section{Conclusion}

L'alliance constitue un mode d'internationalisation efficace pour les PME. Ces dernières ont intérêt à coopérer avec leurs concurrents pour faire face aux obstacles à l'export. La confiance entre les membres de l'alliance s'instaure d'autant plus que la coopération existe entre les alliés. Cela permet de développer des actions concurrentielles grâce à l'apprentissage en collectif et in-fine, la réalisation des objectifs à l'international. 


\section{References:}

1. Arrow, K. (1968). The Economics of Moral Hazard : Further Comment. American Economic Review. pp. 537-539.

2. Arteaga-Ortiz, J. \& Fernández-Ortiz, R. (2008). Des obstacles à l'exportation chez les petites et moyennes entreprises: Une proposition intégrative. Revue internationale PME. Volume $21 . \mathrm{N}^{\circ} 2$. pp. 9-42.

3. Balambo, M. A. \& Baz, J. E. L. (2014). De l'intérêt de l'analyse des modèles des équations structurelles par la méthode PLS dans les recherches sur les relations inter organisationnelles: le cas des recherches en Logistique. In 7ème Edition du colloque international LOGISTIQUA.

4. Barney, J. (1991). Firm resources and sustained competitive advantage. Journal of Management Volume 17. N¹. pp. 99-120.

5. Bengtsson, M. \& Kock, S. (2000). Coopetition in Business Networks - To Cooperate and Compete Simultaneously. Industrial Marketing Management. N²9. pp. 411-426.

6. Bensebaa, F. (2003). La dynamique concurrentielle : défis analytiques et méthodologiques. Finance Contrôle Strategie. Volume 6. $\mathrm{N}^{\circ} 1$. pp. 5-37.

7. Bouncken, R. B. \& al. (2015). Coopetition : A systematic review, synthesis, and future research directions. Review of Managerial Science. pp. 557-601.

8. Brandenburger, A.M. \& Nalebuff, B.J. (1996). Co-opetition. New York : Doubleday.

9. Chtourou, W. (2005). Le développement international des PME : une approche par les réseaux. Revue internationale P.M.E. Volume 18. $\mathrm{N}^{\circ} 1$. pp. 73-102.

10. Dagnino, G. B. \& Le Roy, F. \& Yami, S. (2007). La dynamique des stratégies de coopétition. Revue française de gestion. Volume 176. pp. 87-98.

11. Dagnino, G. B. \& Padula, G. (2002). Coopetition Strategy. A New Kind of Interfirm Dynamics For Value Creation. In Second Annual Conference - Innovative Research in Management.

12. Dussauge, P. \& Garrette, B. (1991). Alliances stratégiques : Mode d'emploi. Revue française de gestion. Volume 85. pp. 4 -18.

13. Fernandes, A. (2012). En quoi l'approche PLS est-elle une méthode à (re)-découvrir pour les chercheurs en management?.M@n@gement, Volume 15. N¹. pp. 102-123.

14. Fernandez, A. \& Le Roy, F. (2010). Pourquoi coopérer avec un concurrent? Une approche par la RBV. Revue française de gestion. Volume 5. N²04. pp. 155-169. 
15. Fornell, C. \& Larcker D. F. (1981). Evaluating Structural Equation Models with Unobservable Variables and Measurement Error. Journal of Marketing Research. Volume 18. $\mathrm{N}^{\circ} 1$. pp. 39- 50.

16. Gnyawali, D.R. \& Park B. J. R. (2011). Co-Opetition between Giants : Collaboration with Competitors for Technological Innovation. Research Policy. Volume 40. N5. pp. 650-663.

17. Gnyawali, R.D. \& He J. \& Madhavan R. (2006). Impact of coopetition on firm competitive behavior : an empirical examination. Journal of Management. Volume 32. $\mathrm{N}^{\circ}$ 4. pp. 507-530.

18. Johanson, J. \& Wiedersheim-Paul, F. (1975). The internationalization of the firm - four Swedish cases. Journal of Management Studies. Volume 12. N³. pp. 305-322.

19. Johanson, J., Vahlne, J. E. (1990). The mechanism of internationalization. International Marketing Review. Volume 7. N4. pp. 11-24.

20. Jolly, D. (2001). Alliances interentreprises, entre concurrence et coopération. Vuibert Entreprendre. Paris.

21. Katsikeas \& Constantine S. \& Leonidou L. C. \& Morgan N. A. (2000). Firm-Level Export Performance Assessment : Review, Evaluation, and Development. Journal of the Academy of Marketing Science. Volume 28. N4. pp. 493-511.

22. Lacroux, A. (2010). L'Analyse Des Modèles De Relations Structurelles Par La Méthode PLS : Une Approche Emergente Dans La Recherche. In XXème congrès de l'AGRH.

23. Leonidou, L. C. \& Katsikeas, C.S. (1996). The export development process : an integrative review of empirical models. Journal of International Business Studies. Volume 27. Third Quarter. pp. 517551.

24. Li, L. \& Li, D. \& et Dalgic, T. (2004). Internationalization Process of Small and Medium-sized Enterprises : Towards a Hybrid Model of Experiential Learning and Planning. Management International Review. Volume 44. N¹. pp. 93-116.

25. Luo, Y. (2004). A co-opetition perspective of MNC-host government relations. Journal of International Management. Volume 10. pp. 431445.

26. Luo, Y. (2005). Toward coopetition within a multinational enterprise : a perspective from foreign subsidiaries. J World Bus. Volume $40 . \mathrm{N}^{\circ} 1$. pp. 71-90.

27. Porter, M. \& Fuller, M. (1986). Coalitions and global strategy. In M. Porter (Eds), Competition in Global Industries, Boston, MA : Harvard Business School Press, pp. 315-344. 
28. Ramadan, M. \& Levratto, N. (2011). Conceptualisation de l'internationalisation des PME. Revue internationale PME. Volume 24. $\mathrm{N}^{\circ} 1$. pp. 71-106.

29. Ramonjavelo, V. \& Préfontaine, L. \& Skander, D. \& Ricard, L. (2006). Une assise au développement des PPP : la confiance institutionnelle, inter organisationnelle et interpersonnelle. Canadian Public Administration. Volume 49. N³. pp. 350-374.

30. Ritala, P. (2012). Coopetition Strategy - When is it Successful? Empirical Evidence on Innovation and Market Performance. British Journal of Management. Volume 23. pp. 307-324.

31. Rutashobya, L. \& JAENSSON, J. E. (2004). Small firms internationalization for developement in Tanzania, Exploring the network phenomenon. International Journal of Social Economics. Volume 31. N¹-2. pp. 159-173.

32. Ruzzier, M. R. \& Hisrich, D. \& Antoncic, B. (2006). SME internationalization research : past, present and future. Journal of Small Business Enterprise Development. Volume 13. $\mathrm{N}^{\circ}$ 4. pp. 476497.

33. Sousa, C. M. P. \& Martínez-López, F. J. \& Coelho, F. (2008). The determinants of export performance : A review of the research in the literature between 1998 and 2005. International Journal of Management Reviews. Volume 10. N4. pp. 343-374.

34. Torrès, O. (2004). Essai de théorisation de la gestion des PME : de la mondialisation à la proxémie. Rapport d'Habilitation à diriger des recherches.

35. Trabelsi, K. (2007). La confiance: la «plate-forme » de l'alliance stratégique. Management \& avenir. Volume 4. $\mathrm{N}^{\circ} 14$. pp. 25-41.

36. Tsai, W. 2002. Social Structure of 'Coopetition' Within a Multiunit Organization : Coordination, Competition, and Intraorganizational Knowledge Sharing. Organization Science. Volume 13. N². pp. 179_ 190.

37. Walley, K. (2007). Coopetition : an introduction to the subject and an agenda for research. Int Stud Manag Org. Volume 37. ํ⒉ pp. 11-31.

38. Xiang, T. (2013). L'impact des coopérations en termes de ressources et d'activités sur la performance : une étude du réseau logistique en Chine. Logistique \& Management. Volume 21. N³. pp. 41-56.

39. Zou, S. \& Stan, S. (1998). The determinants of export performance : a review of the empirical literature between 1987 and 1997. International Marketing Review. Volume 15. N5. pp. 333-356.

40. Zou, S. \& Taylor, C. R. \& Osland, G. E. (1998). The EXPERF Scale : A Cross-National Generalized Export Performance Measure. Journal of International Marketing. Volume 6. N³. pp. 37-58. 